\title{
Empirical Path Loss Model in City-forest Environment for Mobile Communications
}

\author{
Andre A. P. de Carvalho, Iury S. Batalha, Miercio A. Neto,Bruno L. Castro, Fabricio J. B. Barros, Jasmine P. L. \\ Araujo, Gervasio P. S. Cavalcante
}

\begin{abstract}
The feasible choice of a propagation model for a given wireless system depends on environment type among other factors. Thus, it is a crucial decision on radio network planning. This current proposal is a new methodology applied for LTE systems that includes: to find optimal parameters of a propagation model that minimizes Root Mean Square Error (RMSE) and maximizes Grey Relation Grade and Mean Absolute Percentage Error, (GRG-MAPE) in a city-forest environment through the use of metaheuristic optimization such as Cuckoo Search (CS). The results, quantitatively analyzed by RMSE and GRG-MAPE, show a better accuracy of optimized model in comparison with the original version and even with Stanford University Interim (SUI) model.
\end{abstract}

Index Terms-Algorithm Bioinspired, Propagation Model, Channel Modeling, Cuckoo Search, GRG-MAPE

\section{INTRODUCTION}

The birth of LTE (Long Term Evolution) technology, the fourth generation $(4 \mathrm{G})$, and the widespread use of smartphones and tablets, have driven a massive increase in the amount of mobile data traffic. This high use also increased the amount of the studies related to the propagation models for LTE systems, but there is still a gap to take into account. Amazonian characteristics as climate, humidity, afforestation and other factors that are found in such cities-forest that directly influence the received signal level.

Two of the biggest challenges that all network generations have been through are the increase of network usage and the management of different quality of service to the customer. The LTE has successfully overcome both of these challenges. That prioritizes the data traffic instead of voice traffic. The LTE is part of the solution to provide high speed data transfer, being integrated with Wireless Local Area Network as part of the fifth generation (5G) technology [1]. According to specialists, $4 \mathrm{G}$ and $5 \mathrm{G}$ can coexist for a long period of time [2]. Some specific characteristics will help network providers to transition from $4 \mathrm{G}$ to $5 \mathrm{G}$ without impacting customers.

While Evolutionary Algorithms such as Genetic Algorithms, are often used as auxiliary processes to optimize channel modeling. Such methods usually lead to better results than more conventional methods [3], [4]. Evolutionary Algorithms can also be used to help solving other problems in the area related to antennas, georeferencing, and others.

Andre Carvalho, et al. was in Technology Institute, Telecommunication and Computation Laboratory, Belem, PA, Brazil e-mail: andrepcarvalho@gmail.com.

This study was financed in part by the Coordenacao de Aperfeicoamento de Pessoal de Nivel Superior - Brasil (CAPES) - Finance Code 001

Digital Object Identifier: 10.14209/jcis.2021.7
Classic outdoor models present results based on environments with characteristics that are different from the Amazon region. Therefore, parameter adjustments are then required to provide better channel characterization. In order to optimize these adjustments, we propose a new method to find the optimal values for some of these parameters.

Aldossari [5] presents a bibliographic survey is carried out regarding the application of Machine Learning (ML) to assist in modeling and estimating channels. Demonstrating the use of several different techniques for channel estimation. How to use Deep Learning (DL) for estimating the carrier frequency offset, or how to predict the transmitted signals using deep learning techniques such as neural networks other than the classical method where existing receivers estimate the parameters and then recover the data using estimation. Demonstrates that it is possible to use neural network methods such as learned denoising based approximate message passing network to estimate and learn, channel state information, and solve the limited number of frequency chains in cellular. Showing that machine learning techniques are starting in the area of channel modeling to improve efficiency.

In [6] proposes a new ML channel modeling procedure for 5G systems design. ML techniques, particularly supervisory supervision methods, are used to predict the path loss of the wireless channel, a key component of channel modeling. While in [7] demonstrates how artificial intelligence and machine learning combined is applied in the design of $5 \mathrm{G}$ networks to solve unstructured problems and how these techniques can influence channel estimation for network optimization.

In [8] presents an analysis of a hybrid neural network based on error correction for predicting path loss in the frequency range between $800 \mathrm{MHz}$ and $2600 \mathrm{MHz}$, through a combination of propagation models (Okumura Hata, TR 36.942, ECC33, and Ericsson 9999) and an artificial neural network. The results showed Root Mean Square Error (RMSE) indices with greater similarity with the measurements performed in Natal, Rio Grande do Norte, Brazil.

In [9] presents the comparison between traditional channel models to a channel model obtained using DL techniques, using satellite images aided by a simple path loss model. Through experimental measurements at the frequencies of 811 $\mathrm{MHz}$ and $2630 \mathrm{MHz}$, the DL is able to improve the path loss prediction in unmeasured locations. However, in [10] presents a deep convolutional neural network-based approach to estimate channel parameters, using two dimensions satellite images. The experimental results show that the exponent of path loss is $86 \%$ accurate and $76 \%$ accurate for the large-scale 
shading factor, at the frequency of $900 \mathrm{MHz}$.

Ayadi et al. [11] present and evaluate a new propagation model for heterogeneous networks. The designed model is multi-band, multi-environment, and is usable for short and long distance. For this research, a measurement campaign was conducted in Tunis (Tunisia) using continuous wave analog technology. It concerns the most used bands (between 450 and $2600 \mathrm{MHz}$ ) in rural, suburban, and urban environments.

In [12] a study was carried out to determine the most suitable neural network parameters for predicting path loss in the Very High Frequency (VHF) band. The measurements studied were 189.25 MHz and 479.25 MHz. Experiments were performed and the performance of the developed models of Artificial Neural Network are evaluated using the following statistical metrics: Mean Absolute Error, Mean Square Error, Mean Mean Square Error, Standard Deviation and regression coefficient. The results showed that the Artificial Neural Network (RNA) model produced the best performance, and the comparative analysis of the model developed with the Hata, COST 231, ECC-33 and Egli models showed that the RNA-based path loss model has better prediction accuracy and generalization than empirical models.

In [13] a new path loss prediction model is developed based on an Adaptive Neuro-Fuzzy Inference System (ANFIS) for multi-transmitter radio propagation scenarios and applicable to VHF bands. Through measurements collected at the frequencies $89.3 \mathrm{MHz}, 103.5 \mathrm{MHz}$ and $203.25 \mathrm{MHz}$, it was demonstrated by the results that the path loss model based on ANFIS obtained low values for RMSE, and in similar scenarios, the ANFIS model demonstrated good generalizability with RMSE values.

The main contributions of this article are: (1) A measurement campaign in real environments (in Amazonian environments); (2) The use of Bioinspired Computing to optimize large-scale propagation models; (3) Calculation of optimal values for models to better characterize the channel; (4) Adjustments to the propagation model for the Amazon region; and (5) A new methodology for the optimization of propagation models.

The main goal of our contribution is to propose a new methodology to aid in the planning of LTE networks in forested cities with a tropical rainforest climate, improving prediction accuracy in the antenna coverage area. The parameters of Federal University of Para (UFPA) Model [14] were tuning using Cuckoo Search (CS) Metaheuristic Optimization [15]. The results were analyzed and validated comparing with original parameters of UFPA model and a well known model used to compare their behavior for the same propagation scenario, taking into account the distance between transmission and receiver. This other classical model compared with predictions made using UFPA propagation model is called Stanford University Interim (SUI) model [16].

For better understanding, this article is divided into 5 sections. In section 1, a brief introduction with motivation, related works, and scenario of the $4 \mathrm{G}$ technology is described; in section 2, the classical propagation model developed for the Amazonian environment is briefly described; section 3 describes the methodology, measured scenario (Belem-Pa,
Brazil) and implemented algorithm; section 4 describes the results obtained through the methodology developed, and finally section 5 describes the final considerations.

\section{PROpagation MODEL}

The UFPA propagation model has been created to be used in the frequency range of $5.8 \mathrm{GHz}$, and based on path loss measurements collected in 12 different cities in Northern Brazil. Therefore, this model was selected for adjustments in this work. The environment has suburban characteristics, including wooded environments mixed with residential and commercial buildings. However, adjustments to the equation must be made for measurements found in the city of Belem, in the $2.6 \mathrm{GHz}$ frequency range. This model was proposed in [14], provided by

$$
\begin{aligned}
& L=K_{1} * \log _{10}(d)+K_{2} * \log _{10}(f * 1 e-6) \\
& +a-b * \frac{\left(\left(h_{t}+h_{r}\right) * 3 e 8 / f\right)}{\left(0,1 * H_{o b}\right)}
\end{aligned}
$$

where:

$d$ - Distance between transmitter and receiver

$f$ - Frequency

$K_{1}, K_{2}$ - Parameters obtained with linear least squares

$a, b$ - Parameters adjusted by linear least squares

$h_{r}$ - Receiver Antenna Height

$h_{t}$ - Height of Transmitting Antenna

$H_{o b}$ - Average obstructions height.

The parameters $K_{1}, K_{2}, a$, and $b$ cited in the equation (1) will be adjusted for the $2.6 \mathrm{GHz}$ frequency range. This adjustment will be done through Cuckoo Search Optimization Algorithm. As the height of the obstructions is fixed, an average height of 62 meters was adopted. The height of the transmitting antenna is 40 meters and the height of the receiver is 1.60 meters.

\section{Methodology}

The methodology is organized as follows: scenario of measurements, measurement campaign and data treatment, finally the cuckoo search algorithm description applied to propagation model adjustment.

\section{A. Scenario of Measurement}

The scenario of measurements is the city of Belem, which is located on the equator where all four seasons are not clearly defined during the year (summer, fall, winter and spring). Due to the geographic location Belem has actually only a period with little rain and another period with much rain. The city is bathed by a freshwater river and this influences the relative humidity of the air which most of the time is very high.

The capital of the state of Para is an arboreus amazonian town and, as previously stated, bathed by a river. In the routes where were made the measurements it is possible to find tunnels of trees, so is possible to check the influence of vegetation and architecture on the power received. 


\section{B. Measurement Campaign}

It was carried out on Belem streets, from the Federal University of Para with an average speed of $30 \mathrm{~km} / \mathrm{h}$, considering the height of the receiver of $1.60 \mathrm{~m}$, under the night. During the measurement campaign the variables of wind, temperature and relative humidity of the air were monitored, where on average the wind was gusting to $6 \mathrm{~km} / \mathrm{h}$, the minimum temperature of $24^{\circ} \mathrm{C}$ and maximum of $30^{\circ} \mathrm{C}$ and finally the relative humidity of the air in $96 \%$.

The antenna installed and used in the work from operates in the frequency: $2.6 \mathrm{GHz}$; Power: 40 watts; Gain: $15.4 \mathrm{~dB}$; Height: 40 meters; Latitude: -1.46425 ; Longitude: -48.44905 ; Environment: wooded and flat. The company operator is the responsible for the antenna studied in this work.

A Fig. 1 demonstrates the selected routes from the mapped streets. The geographical position of the antenna is shown with the measured points. The selected routes had as main feature the afforestation present in the city, which is similar to other cities in the Amazon region. For each route were carried out at least 2 turns of measurements. The antenna is located near the UFPA's Environmental Park .

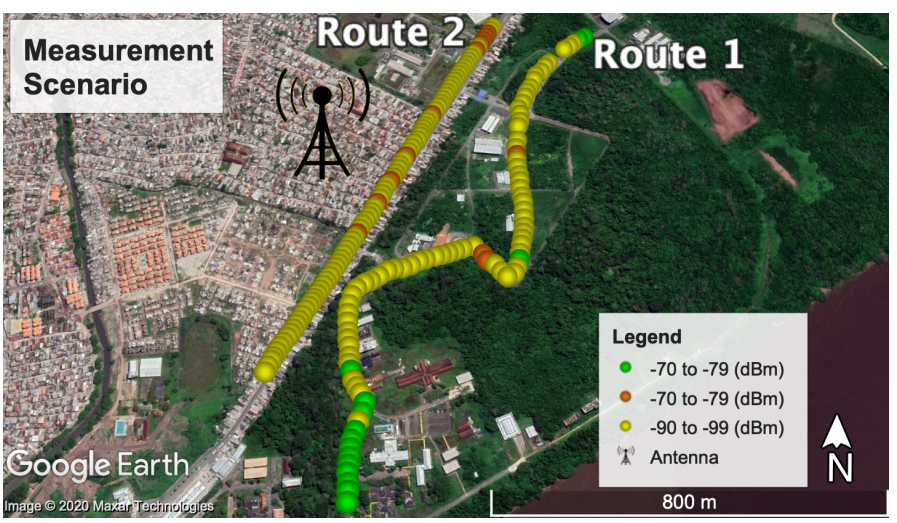

Fig. 1. Measurement Route

\section{Cuckoo Search}

For simplicity in describing the Cuckoo Search (CS) metaheuristic optimization technique [17], let's consider the following three idealized rules: 1) Each cuckoo lays one egg at a time, and dump its egg in a randomly chosen nest; 2) The best nests with high quality of eggs will carry over to the next generations; 3) The number of available host nests is fixed, and the egg laid by a cuckoo is discovered by the host bird with a probability $p_{a} \in[0,1]$.In this case, the host bird can either throw the egg away or abandon the nest, and build a completely new nest. For simplicity, this last assumption can be approximated by the fraction $p_{a}=0.25$ of the $n=100$ nests are replaced by new nests (with new random solutions). For a maximization problem, the quality or fitness of a solution can simply be proportional to the value of the fitness function.

Other forms of fitness can be defined in a similar way to the fitness function in genetic algorithms. For simplicity, we can use the following simple representations that each egg in a nest represents a solution, and a cuckoo egg represents a new solution; the aim is to use the new and potentially better solutions (cuckoos) to replace a not-so-good solution in the nests. Of course, this algorithm can be extended to the more complicated case where each nest has multiple eggs representing a set of solutions. For this present work, we will use the simplest approach where each nest has only a single egg.

In the application of the cuckoo search optimization in the definition of best parameter values for the UFPA propagation model, the description of the proposed implementation and used in this work is based on the initial algorithm described in the previous paragraphs and published by Xin She Yang [15]:

Step 1 - The first nests / solutions start with random values of $K_{1}, K_{2}, a$ and $b$. There are 100 nests / solutions generated.

Step 2 - The fitness of these 100 proposals of nests / solutions is then calculated and the best one is saved.

Step 3 - The algorithm then follows the execution by entering the loop of iterations that was defined as 1000 .

Step 3.1 - Generate new nests / solutions and evaluation of the best among them.

Step 3.2 - New nests / solutions are then generated again and the probability of finding alien eggs $\left(p_{a}\right)$ is applied so some nests / solutions are replaced by other random values. This set of nests / solutions is then evaluated. The best of them is kept. Step 3.3 - Increase the loop counter and go back to the beginning of it.

Step 4 - At the end of the 1000 iterations, you have the best nest / solution found by the cuckoo search algorithm with best $K_{1}, K_{2}, a$ and $b$ values that will fit better with measurements data.

The fitness function is based on the UFPA propagation model, i.e., $K_{1}, K_{2}, a$ and $b$ are used from the nests/solutions and compared with the path loss measurement at the same points. A root mean square error is calculated between both and the nest/solution that generate the lowest RMSE is kept as the best ones.

\section{REsults}

In this section will be demonstrated the results obtained through the developed methodology. Orange color always represents the data processed in the UFPA original propagation model (with no adjustments); the yellow color represents the data processed with adjustment using CS algorithm. Route 1 is inside the park and Route 2 is outside the park, where this route has characteristics of a city-forest in the Amazon region, Fig. 2 shows Route 1 and Route 2 optimized.

The comparison of the results found with classic SUI model and default UFPA model is done through metrics RMSE and Grey Relation Grade and Mean Absolute Percentage Error (GRG-MAPE). The RMSE and GRG-MAPE are metrics of evaluation and performance, the most used to measure the accuracy of predictive models that target continuous variables. Where RMSE is the measure that calculates the mean square root of the errors between observed values (reals) and predictions (hypotheses). While to the GRG-MAPE [18] ranged from 0 to 1 represents matching degree between estimation data and standard data. 

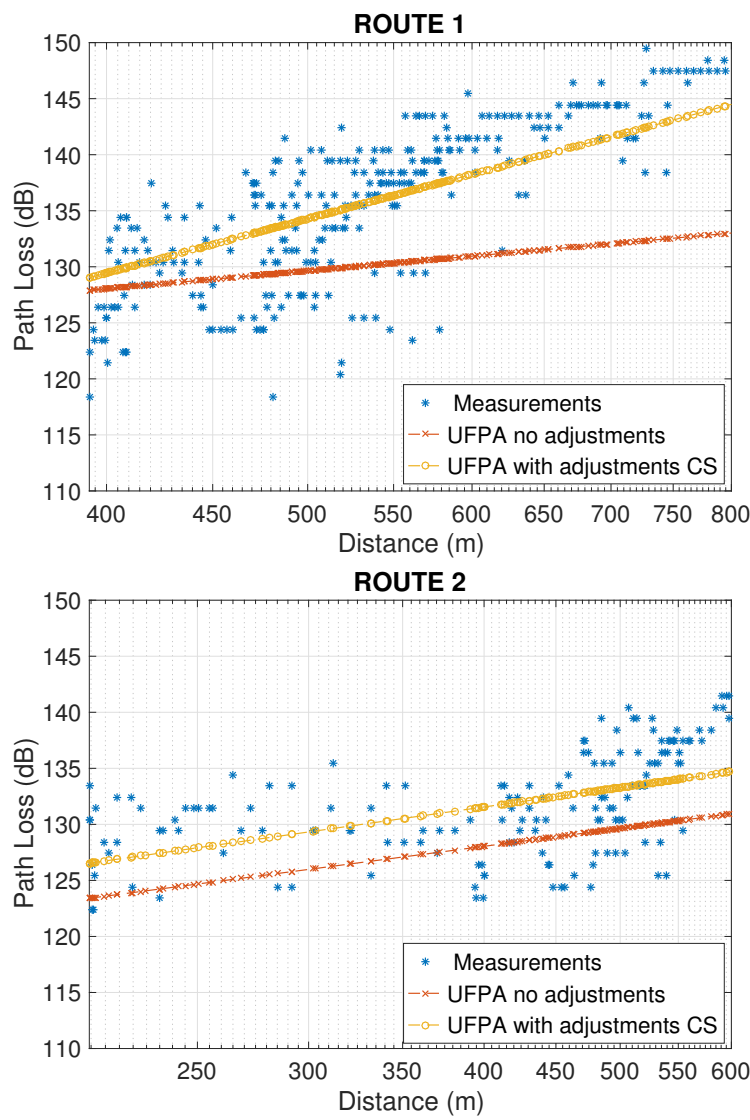

Fig. 2. Measurement Routes

TABLE I

COMPARISON OF RMSE AND GRG-MAPE TWO ROUTES

\begin{tabular}{|l|l|l|l|}
\hline & & & UFPA \\
Route & SUI & UFPA & Optimized \\
\hline \multicolumn{4}{|c|}{ RMSE (dB) } \\
\hline Route 1 & 23.30 & 8.38 & 4.86 \\
\hline Route 2 & 22.08 & 7.67 & 6.54 \\
\hline \multicolumn{4}{|c|}{ GRG-MAPE (dB) } \\
\hline Route 1 & 0.80 & 0.82 & 0.87 \\
\hline Route 2 & 0.79 & 0.84 & 0.85 \\
\hline
\end{tabular}

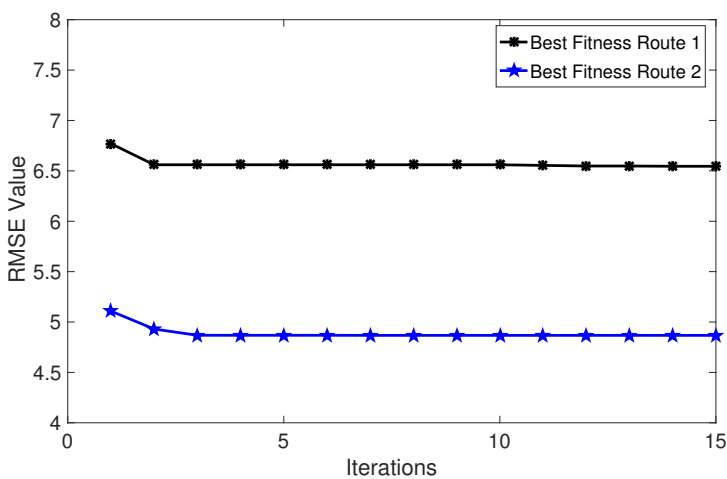

Fig. 3. Convergence of aptitude function of Cuckoo Search algorithm

The UFPA Model proved to be the best model for Tropical rainforest climate cities according to Table I, and at this work it was validated its use in the $2.6 \mathrm{GHz}$ range besides $5.8 \mathrm{GHz}$ through parameters.

Analyzing the results obtained in the RMSE and GRGMAPE tables, it is possible to verify that the UFPA optimized model presents the lowest RMSE and highest GRG-MAPE with improvements in accuracy prediction as showed in Table I and Fig. 3. Moreover, the convergence of the CS algorithm in the optimization process of the UFPA propagation model went rapidly from 6.77 to $6.55 \mathrm{~dB}$ after only about 10 iterations for route 1 , and for route 2 from 5.1 to $4.87 \mathrm{~dB}$ after only 3 iterations.

\section{CONClusion}

This work presented a methodology for adjusting propagation model for city-forest environments. With the results of the original propagation model (without any adjustment) and with adjustment done by bioinspired algorithm, the latter proved to be more accurate than the other one and also better when compared with a classical propagation model used at this frequency range studied, i.e, $2.6 \mathrm{GHz}$, called SUI model.

The use of prediction methods through adjustments with bioinspired metaheuristic algorithms in the planning phase of the communication systems is aimed at increasing the efficiency and accuracy in several environments of base station deployment, also representing time and money saving for those operating in these scenarios. Therefore, this new technique is a useful tool for LTE and LTE-A wireless network engineers, proving to be an effective and accurate methodology.

The main emphasis was on the optimizing parameters of UFPA model. The results showed a feasible curve for a Tropical rainforest climate region, specifically at the cityforest scenario. Moreover, UFPA model was proposed to attend $5.8 \mathrm{GHz}$ frequency range at densely wooded suburban environments presented at Amazonian cities, then with the optimized adjustments using metaheuristic optimization this work extends the model to attend $2.6 \mathrm{GHz}$ frequency range with the best accuracy presented at Table I.

It is worthwhile to highlight that the adjusted UFPA model to Belem, Brazil, using field measurements done at outdoor environments and bioinspired CS technique, exhibit an improvement in its accuracy, in terms of RMSE and GRGMAPE.This proposed methodology can be applied for any telecommunication system and any measurement environment.

\section{ACKNOWLEDGMENT}

We thank our teachers, friends, and all those who contributed directly and indirectly to the success of this work, in particular to the Institute of Technology of UFPA and the Laboratory of Computation and Telecommunications.

\section{REFERENCES}

[1] S. Sirotkin, LTE-WLAN aggregation (LWA): benefits and deployment considerations, Intel corporation white paper, 2017. https://www.intel.com/content/dam/www/public/us/en/documents/whitepapers/lte-wlan-aggregation-deployment-paper.pdf

[2] C. Huang and W. Lin, A radio transceiver architecture for coexistence of 4G-LTE and $5 G$ systems used in mobile devices, in IEEE MTT-S International Microwave Symposium (IMS), 2017. doi: 10.1109/MWSYM.2017.8059075. 
[3] M. Feng, L. Guomin and G. Wenrong. Heterogeneous Network Resource Allocation Optimization Based on Improved Bat Algorithm, in International Conference on Sensor Networks and Signal Processing (SNSP), 2018. doi: $10.1109 /$ SNSP.2018.00020

[4] E. H. Houssein, M. R. Saad, K. Hussain, W. Zhu, H. Shaban and M. Hassaballah, Optimal Sink Node Placement in Large Scale Wireless Sensor Networks Based on Harris Hawk Optimization Algorithm, in IEEE Access, volume: 8. 2020. doi: 10.1109/ACCESS.2020.2968981

[5] S. M. Aldossari, KC. Chen, Machine Learning for Wireless Communication Channel Modeling: An Overview. Wireless Pers Commun 106, 4170 (2019). doi: https://doi.org/10.1007/s11277-019-06275-4

[6] S. Aldossari, K. Chen, Predicting the Path Loss of Wireless Channel Models Using Machine Learning Techniques in MmWave Urban Communications, 2019 22nd International Symposium on Wireless Personal Multimedia Communications (WPMC), Lisbon, Portugal, 2019, pp. 1-6, doi: 10.1109/WPMC48795.2019.9096057.

[7] C. Wang, M. D. Renzo, S. Stanczak, S. Wang and E. G. Larsson, Artificial Intelligence Enabled Wireless Networking for $5 G$ and Beyond: Recent Advances and Future Challenges, in IEEE Wireless Communications, vol. 27, no. 1, pp. 16-23, February 2020, doi: 10.1109/MWC.001.1900292.

[8] B. J. Cavalcanti, G. A. Cavalcante, L. M. de Mendona, G. M. Cantanhede, M. M.M. de Oliveira, A. G. DAssuno A Hybrid Path Loss Prediction Model based on Artificial Neural Networks using Empirical Models for LTE And LTE-A at $800 \mathrm{MHz}$ and $2600 \mathrm{MHz}$, J. Microw. Optoelectron. Electromagn. Appl. vol.16 no.3 So Caetano do Sul Sept. 2017. doi: https://doi.org/10.1590/2179-10742017v16i3925

[9] J. Thrane, D. Zibar and H. L. Christiansen, Model-Aided Deep Learning Method for Path Loss Prediction in Mobile Communication Systems at 2.6 GHz, in IEEE Access, vol. 8, pp. 7925-7936, 2020, doi: 10.1109/ACCESS.2020.2964103.

[10] H. F. Ates, S. M. Hashir, T. Baykas and B. K. Gunturk, Path Loss Exponent and Shadowing Factor Prediction From Satellite Images Using Deep Learning, in IEEE Access, vol. 7, pp. 101366-101375, 2019, doi: 10.1109/ACCESS.2019.2931072.

[11] M. Ayadi, A. B. Zineb, S. Tabbane, A UHF path loss model using learning machine for heterogeneous networks, IEEE Transactions on Antennas and Propagation 65 (7) (2017) 36753683. doi:10.1109/TAP.2017.2705112.

[12] S. I. Popoola et al. Determination of Neural Network Parameters for Path Loss Prediction in Very High Frequency Wireless Channel, in IEEE Access, vol. 7, pp. 150462-150483, 2019, doi: 10.1109/ACCESS.2019.2947009.

[13] T. Nazmat, Surajudeen-Bakinde, N. Faruk, S. I. Popoola, M. A. Salman, A. A. Oloyede, L. A. Olawoyin, C. T. Calafate. Path loss predictions for multi-transmitter radio propagation in VHF bands using Adaptive Neuro-Fuzzy Inference System, Engineering Science and Technology, an International Journal, Volume 21, Issue 4, 2018, Pages 679-691, ISSN 2215-0986, doi: https://doi.org/10.1016/j.jestch.2018.05.013.

[14] W. D. Meza, G. Siqueira, L. J. Matos, et al., Channel characterization in the $5.8 \mathrm{ghz}$ band in a suburban area, Journal of Microwaves, Optoelectronics and Electromagnetic Applications 13 (1) (2014) 97110 doi: https://doi.org/10.1590/S2179-10742014000100008

[15] X. S. Yang, S. Deb, Cuckoo search via levy flights, in: Proc. World Congress Nature Biologically Inspired Computing (NaBIC), 2009, pp. 210214. doi:10.1109/NABIC.2009.5393690.

[16] V. Erceg, L. J. Greenstein, S. Y. Tjandra, S. R. Parkoff, A. Gupta, B. Kulic, A. A. Julius, R. Bianchi, An empirically based path loss model for wireless channels in suburban environments, IEEE Journal on Selected Areas in Communications 17 (7) (1999) 12051211. doi: https://doi.org/10.1109/49.778178

[17] X.-S.Yang,A new metaheuristic bat-inspired algorithm. (arXiv:1004 4170v1) https://arxiv.org/abs/1004.4170

[18] J. Yu, C. Li, K. Yang and W. Chen GRG-MAPE and PCC-MAPE Based on Uncertainty-Mathematical Theory for Path-Loss Model Selection, 2016 IEEE 83rd Vehicular Technology Conference (VTC Spring), Nanjing, 2016, pp. 1-5, doi: 10.1109/VTCSpring.2016.7504265. 